\title{
Dosing parameters for the effects of high- frequency transcranial magnetic stimulation on smoking cessation: study protocol for a randomized factorial sham-controlled clinical trial
}

Ellen Carl ${ }^{1 *}$, Amylynn Liskiewicz', Cheryl Rivard ${ }^{1}$, Ronald Alberico ${ }^{1}$, Ahmed Belal ${ }^{1}$, Martin C. Mahoney ${ }^{1}$, Amanda J. Quisenberry' ${ }^{1}$ Warren K. Bickel ${ }^{2}$ and Christine E. Sheffer ${ }^{1}$

\begin{abstract}
Background: Despite the considerable success of comprehensive tobacco control efforts, tobacco use remains one of the greatest preventable causes of death and disease today. Over half of all smokers in the US make quit attempts every year, but over 90\% relapse within 12 months, choosing the immediate reinforcement of smoking over the long-term benefits of quitting. Conceptual and empirical evidence supports continued investigation of high frequency repetitive transcranial magnetic stimulation (rTMS) of the left dorsolateral prefrontal cortex in reducing relapse and decreasing cigarette consumption. While this evidence is compelling, an optimal dosing strategy must be determined before a long-term efficacy trial can be conducted. The goal of this study is to determine a dosing strategy for $20 \mathrm{~Hz}$ rTMS that will produce the best long-term abstinence outcomes with the fewest undesirable effects.

Methods: This is a fully crossed, double-blinded, sham-controlled, $3 \times 2 \times 2$ randomized factorial study. The three factors are duration (stimulation days: 8, 12, and 16); intensity (900 or 1800 pulses per day); and sham control. Participants $(n=258)$ will consist of adults $(18-65)$ who are motivated to quit smoking cigarettes and who will be followed for 6 months post-quit. Outcomes include latency to relapse, point prevalence abstinence rates, delay discounting rates, cognitive-behavioral skills acquisition, and multiple measures of potential undesirable effects that impact participant compliance.

(Continued on next page)
\end{abstract}

\footnotetext{
* Correspondence: Ellen.Carl@roswellpark.org

${ }^{1}$ Roswell Park Comprehensive Cancer Center, Buffalo, NY 14203, USA

Full list of author information is available at the end of the article
}

(C) The Author(s). 2020 Open Access This article is licensed under a Creative Commons Attribution 4.0 International License, which permits use, sharing, adaptation, distribution and reproduction in any medium or format, as long as you give appropriate credit to the original author(s) and the source, provide a link to the Creative Commons licence, and indicate if changes were made. The images or other third party material in this article are included in the article's Creative Commons licence, unless indicated otherwise in a credit line to the material. If material is not included in the article's Creative Commons licence and your intended use is not permitted by statutory regulation or exceeds the permitted use, you will need to obtain permission directly from the copyright holder. To view a copy of this licence, visit http://creativecommons.org/licenses/by/4.0/. The Creative Commons Public Domain Dedication waiver (http://creativecommons.org/publicdomain/zero/1.0/) applies to the data made available in this article, unless otherwise stated in a credit line to the data. 
(Continued from previous page)

Discussion: This study integrates existing theoretical concepts and methodologies from neuropsychology, behavioral economics, brain stimulation, clinical psychology, and the evidence-based treatment of tobacco dependence in the development of a promising and innovative approach to treat tobacco dependence. This study will establish an optimal dosing regimen for efficacy testing. Findings are expected to have a significant influence on advancing this approach as well as informing future research on clinical approaches that combine rTMS with other evidence-based treatments for tobacco dependence and perhaps other addictions.

Trial registration: Clinical Trials NCT03865472 (retrospectively registered). The first participant was fully enrolled on November 26, 2018. Registration was posted on March 7, 2019.

Keywords: Smoking cessation, Tobacco dependence, Transcranial magnetic stimulation, Relapse prevention, Brain stimulation, Neuromodulation, Delay discounting, Dorsolateral prefrontal cortex

\section{Background}

Despite the considerable success of comprehensive tobacco control efforts, tobacco use remains one of the greatest preventable causes of death and disease today [1]. In the US alone, cigarette smoking causes $>480,000$ deaths annually including a third of all cancer deaths, and $87 \%$ of all lung cancer deaths [1, 2]. Most smokers want to quit and over half make a quit attempt every year, but over $90 \%$ choose the immediate reinforcement of smoking over the significant long-term benefits of quitting [3-7] within 12 months. This conundrum of relapse to smoking despite considerable efforts among smokers to quit continues to be one of the most significant public health challenges today $[1,2]$.

Smoking cessation involves repeatedly choosing between the known, immediate, and reinforcing experience of smoking and a variety of dynamic choice options from which the smoker repeatedly chooses throughout the day in the context of continually fluctuating neurobiological, environmental, and cognitive influences [8-10]. Executive functions such as self-control are essential to managing the repetitive choice process if one is to attain long-term abstinence [11-13]. The dorsolateral prefrontal cortex (dlPFC), a functional node in the prefrontal cortex (PFC), has a significant role in self-control in general and the controlled response inhibition associated with managing the desire to smoke, called incentive salience [14-19]. Executive function neural processing deficits among smokers parallel decreased activity in the PFC [20] and compromise smokers' ability to manage incentive salience and maintain the choice to quit smoking [21, 22]. Moreover, the absence of nicotine during a quit attempt amplifies executive function neural processing deficits, increases incentive salience, and is likely to contribute to the choice to smoke, even if it means relapse [23, 24].

Chronic nicotine use modifies multiple structures in the endogenous reward system of the brain $[25,26]$. The pleasant and remarkably reinforcing experience of nicotine administration is associated with excessive neurochemical activity in limbic and paralimbic regions of the brain, particularly the ventral tegmental area, nucleus accumbens, and amygdala. In addition, conditioning links previously neutral stimuli with this reinforcing experience creating powerful cues that trigger increases in incentive salience. Over time, this system becomes dysregulated and associated with increased impulsive use of nicotine and decreased sensitivity to natural rewards (e.g., food, water, sex, nurturing) as well as significant executive function neural processing deficits [20, 23, 24, 27].

The Competing Neurobehavioral Decision Systems (CNDS) Model is a well-established dual-systems guide to conceptualizing the psychological processes involved with self-control (e.g., healthy, prudent, far-sighted decisions) in the context of immediately rewarding and less healthy choices (e.g., smoking) [28-30]. The CNDS model posits that decisions are driven by the interaction between two functional neurobiological networks: The executive function network, embodied in the PFC; and the impulsive network, embodied in the limbic and paralimbic regions of the brain [31-35]. Numerous behavioral, neuroimaging, and other studies support this contention [31-34, 36]. Greater PFC activity is linked with a higher likelihood of choosing more prudent, larger later rewards, even in the context of temptation [37]. Direct and indirect manipulation of the PFC appears to alter impulsive decision-making [30].

Among humans and animals, the value of reinforcers is discounted as a function of the time to receipt [38]. Most of us prefer immediate reinforcement but we are willing to wait for larger rewards to varying degrees [39]. The degree to which one discounts, or de-values, delayed reinforcers is called the delay discounting rate [40-43]. Discounting has been applied to a variety of commodities (i.e., money, health, cigarettes, drugs, etc.), magnitudes ( $\$ 5, \$ 100, \$ 1000$, etc.), and signs (i.e., gains, losses) [44]. Importantly, smokers consistently demonstrate higher discounting rates than non-smokers [44-49] and higher discounting rates among smokers are associated with a greater propensity for relapse [34, 50-54]. Discounting rates also reliably decrease with effective addictions 
treatment $[35,52,53]$. Anatomical and functional evidence indicates that delay discounting rates are associated with relative activity levels in two frontal-striatal neural circuits that parallel the functional neurobiological networks in the CNDS $[31,55,56]$. Imaging studies show that higher discounting rates are associated with decreased activity in the PFC relative to limbic/paralimbic activity $[32,56]$. Delay discounting rate is now considered a biological marker for the relative functioning of the two networks described in the CNDS model [28, 30, 35, 57].

Repetitive transcranial magnetic stimulation (rTMS) is a non-invasive brain stimulation technique that selectively modulates neuronal activity by generating electrical currents produced by an electromagnetic coil [58-62]. When applied repetitively in consecutive pulses, rTMS can have a long-term impact on neural circuits [63]. High Frequency (HF) rTMS $(>1 \mathrm{~Hz})$ of the PFC increases regional cerebral blood flow and cortical excitability and improves cognitive function, attention, learning, and memory [64-69]. rTMS has been FDA approved for the treatment of medication resistant unipolar major depressive disorder since 2008 [70]. Neuromodulation of the dlPFC with rTMS affects discounting rates [71, 72], and other types of impulsive and risky decision-making [19, 73-76]. In a preliminary study, 1 session (900 pulses) of HF rTMS (10 or $20 \mathrm{~Hz})$ of the left dIPFC decreased delay discounting rates with $20 \mathrm{~Hz}$ rTMS demonstrating significantly larger effects on delay discounting rates than $10 \mathrm{~Hz}[10]$.

HF rTMS has demonstrated some potential for reducing cigarette consumption and craving in multiple studies [77-79]. Inconsistent findings among these studies is likely associated with variability in frequency $(10-20 \mathrm{~Hz})$, variability in the number of stimulation sessions (range 1-10), a lack of behavioral treatment components, variability in motivation to quit among participants, variability in stimulation site targeting method, variability in the assessment of craving, variability in procedures, and variability in the use of well-established abstinence outcome measures. HF rTMS of the PFC also has been shown to improve learning, memory, attention, and working memory as well as increase regional cerebral blood flow in addition to improving connectivity in the executive function network and enhancing working memory performance [64, 68, 80-82]. Proposed mechanisms of change include increasing long-term potentiation (LTP) and enhancing fronto-striatal pathway connectivity and dopamine function $[83,84]$. LTP is one of several mechanisms involved with increasing synaptic plasticity by enhancing neuronal signal transmission $[60,66,85]$. LTP, through modifications of synaptic strength, appears to be one of the major cellular mechanisms that underlie learning and memory [86]. Finally, a systematic review of rTMS depression treatment studies suggests that
rTMS of the PFC might be a promising technique for cognitive enhancement [87].

Many of the cognitive processes that comprise executive function are mediated by the dIPFC and are also important to cognitive behavioral skill acquisition. A cognitivebehavioral approach to the treatment for tobacco dependence promotes understanding of the cue-urge-response decision-making cycle, awareness of a multitude of conditioned internal and external cues encountered in daily life, and the development of choice options and strategies to manage incentive salience $[88,89]$. Across the treatments of disorders, cognitive-behavioral treatment incorporates learning elements of self-control over one's thoughts, behaviors, and feelings as reactions and emotions are monitored and thoughts are restructured (cognitive restructuring) as well as learning to activate particular behaviors in a range of contexts through anticipation of high-risk situations and planning (behavioral activation) $[88,90]$. These acquired skills include self-monitoring, impulse control, stimulus control, stress management, and problem solving, etc. Cognitive-behavioral approaches can be delivered in varying intensities from self-help to intensive group or individual treatment, but all evidence-based "counseling" interventions for tobacco dependence are based on cognitive-behavioral principles $[88,89,91]$ so we have included a cognitive-behavioral treatment in the current study.

Combining rTMS with cognitive-behavioral treatment has been shown to be feasible and therapeutically promising in the treatment of major depression [91], posttraumatic stress disorder [92], cognitive rehabilitation [93], and relapse to smoking [94]. The effects of HF rTMS of the PFC on learning, memory, and attention suggests that combining HF rTMS of the dIPFC with cognitive-behavioral interventions might facilitate the acquisition of cognitive-behavioral skills, enhance the overall effectiveness of the cognitive-behavioral intervention, and ultimately contribute to improved abstinence outcomes.

To address these limitations, we conducted a feasibility study that combined 8 sessions (900 pulses each session) of $20 \mathrm{~Hz}$ rTMS of the left dIPFC with an evidence-based cognitive-behavioral self-help intervention in a doubleblind randomized sham-controlled trial with treatmentseeking smokers $(n=29)$ and multiple well-established tolerability and abstinence measures. The findings showed potential efficacy. It was found to be feasible and welltolerated, to significantly decrease discounting rates, and to improve multiple abstinence outcomes [94]. In our feasibility study, we combined 8 sessions of $20 \mathrm{~Hz}$ rTMS of the left dIPFC with the a series of 8 evidence-based selfhelp booklets [94]. These 8 booklets, called Forever Free, include multiple, practical cognitive-behavioral skills including understanding and intervening with the cue-urge- 
response cycle and engaging in cognitive restructuring and behavioral activation [95-97]. Treatment-seeking smokers $(n=29)$ who read one new booklet in order per session during the 8 stimulation sessions, were encouraged to continue reading outside sessions, and were followed for 12 weeks. Outcomes included latency to relapse, delay discounting rates, and 12-week, 7-day point prevalence abstinence rates. The mean latency to relapse for active rTMS was 45.19 (SD 9.42) days and sham 20.46 (SD 7.46) days. The median latency to relapse for active rTMS was 33.50 (IQR 7-85) and sham 8.00 (IQR 2 to 37) days. Active rTMS reduced the relative risk of relapse 3fold (RR 0.29, CI 0.10-0.76, Likelihood ratio $\chi^{2}$ with $1 \mathrm{df}=$ $6.40, p=.01)$. Active stimulation also decreased delay discounting of $\$ 100(\mathrm{~F}(1,25.3694)=4.14, p=.05)$ and $\$ 1000$ $(\mathrm{F}(1,25.169)=8.42, p<.01)$, increased 12-week abstinence rates (active $50 \%$ vs. sham $15.4 \%, \mathrm{X}^{2}(\mathrm{df}=1)=3.80$, $\mathrm{p}=.05$ ), and increased uptake of the materials. Side effects included headache and stiffness, but were minimal, resolved quickly, and were well-tolerated. Participants were engaged throughout treatment; 69\% completed all 8 sessions with minimal compensation ( $\$ 10$ per session) and limited appointment availability due to staffing and access to the machine. These findings indicate that this approach has potential long-term efficacy, but more research is needed to determine the optimal dosing regimen to prepare for future efficacy testing.

While the premise and the preliminary evidence for this approach are compelling, an optimal dosing strategy must be determined before a Phase III trial with longterm efficacy outcomes can be conducted [98]. Dose determination studies are often carried out in Phase II of the development of an intervention to ensure that efficacy testing is conducted using the most promising dosing regimens [98]. Because the doses being tested in this protocol have already been found to be safe in many other studies [99-102], safety elements will be monitored, but safety is not the primary focus of this study. We follow a typical plan for a dose determination study by including a sham comparison and a range of lower to higher dosing regimens in an innovative, parsimonious factorial design [98].

The goal of this study is to determine a dosing strategy for $20 \mathrm{~Hz}$ rTMS that will produce the best long-term abstinence outcomes with the fewest undesirable effects. The aims of this study are to examine the effects of stimulation duration (stimulation days) and intensity (pulses per day) on outcomes among smokers $(n=258)$ who are motivated to quit smoking, and to identify the most promising dosing strategy by balancing effect sizes and undesirable effects. The study outcomes include latency to relapse, point prevalence abstinence rates, delay discounting rates, the acquisition of cognitive-behavioral skills, and potential undesirable effects, such as non- compliance and excessive participant burden. The findings will characterize the nature of the rTMS doseresponse relations among two rTMS dosing parameters (e.g., duration and intensity). Our hypotheses are that the duration and intensity will be positively associated with effect size for latency to relapse, abstinence rates, delay discounting rates, and cognitive-behavioral skills acquisition and that the highest duration and intensity doses will be associated with greater levels of undesirable effects (e.g., noncompliance due to participant burden).

\section{Methods}

This study is a fully crossed, double-blind, shamcontrolled, $3 \times 2 \times 2$ randomized factorial design. This factorial design is intended to screen duration and intensity components simultaneously to identify the most promising combination for an optimal dosing intervention for efficacy testing. The factors are duration (stimulation days: 8,12 , and 16), intensity (900 or 1800 pulses per day delivered in 1 and 2 sessions respectively), and a sham control for each condition (see Fig. 1). This study was submitted to, and approved by, Roswell Park Comprehensive Cancer Center's (RPCCC) Institutional Review Board (IRB); all protocol modifications are sent to RPCCC's IRB as well. Prior to completing any studyrelated procedures, participants will sign a written informed consent form. Participants in all conditions will be asked to complete 4 days of stimulation in a 7-day period and those assigned to conditions with 2 sessions per day will have sessions separated by at least $2 \mathrm{~h}$. The days of stimulation must be completed within a specific timeframe (i.e., 8 days of stimulation within 14 days, 12 within 21 days, and 16 within 28 days). The sham control allows for a comparison group to calculate effect sizes while accounting for non-specific treatment-related effects. Participants are followed and assessed every 2 weeks for 24 weeks after initiating rTMS sessions. All inperson research activities will take place at RPCC's campus, inside the Prevention Center building. The Prevention Center has its own parking and is well-suited for use as a research facility.

\section{Power analysis/sample size justification}

Preliminary evidence suggests a mean of 20 days to relapse in the sham groups $(n=11)$. With $n=32$ in the active groups and a common standard deviation of $20 \times$ $0.40=8.0$ across cells, this design has $80 \%$ power to detect a main effect of at least 4 days to relapse in a dichotomous factor (Cohen's $\mathrm{d}=.50 ; 2$-sided $\alpha=0.05, \mathrm{SD}=$ 8.0). With exponentially distributed days to relapse, a 4day change in the mean equates to a detectable hazard ratio of 0.85 or less, in favor of the active condition.

Using the preliminary study as a guide, assuming days to relapse are exponentially distributed, the corresponding 


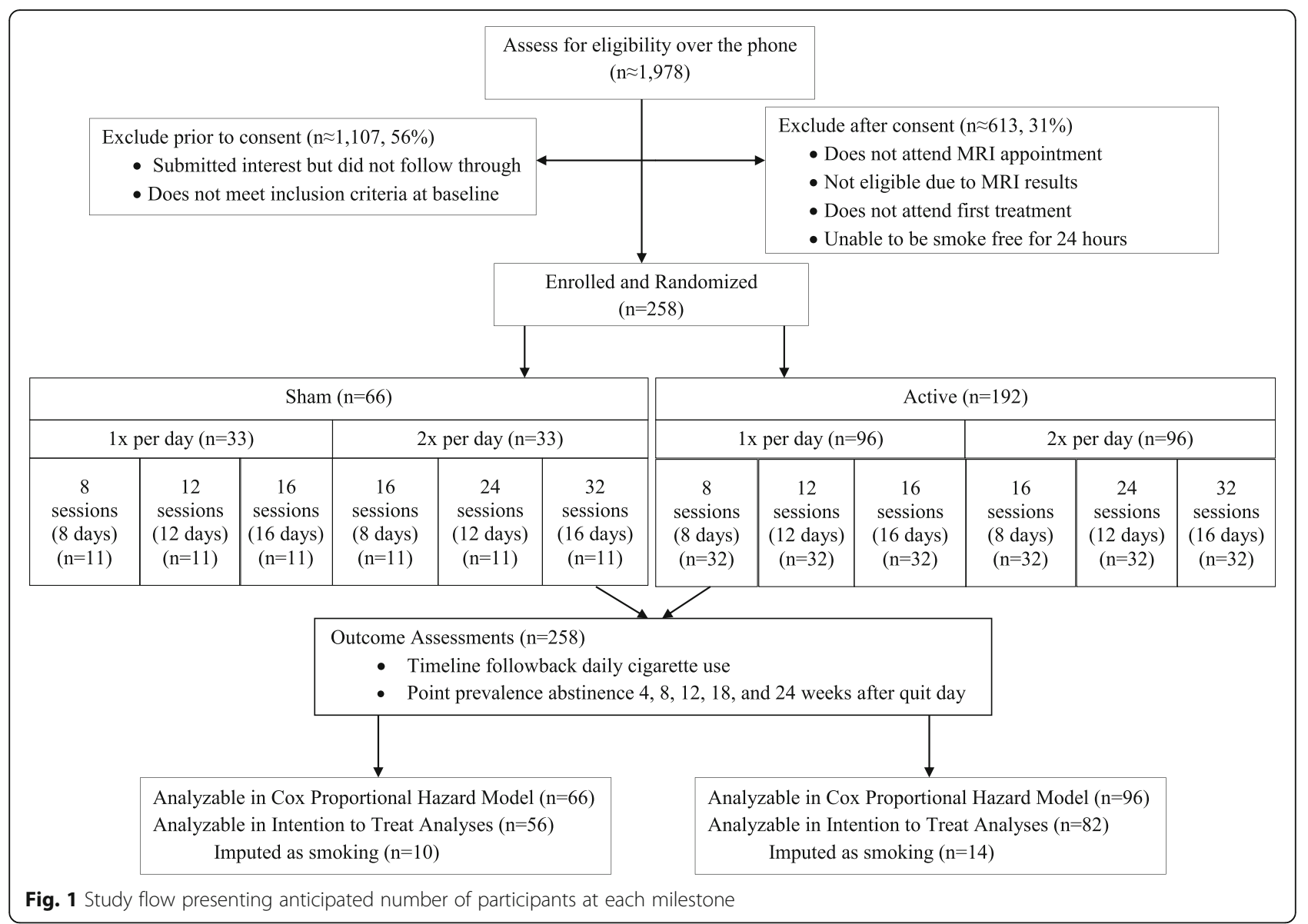

hazard ratio (active vs. sham) is between 0.24 and 0.45 , based on means. This presents a relatively small-medium effect size but significant in the context of a study with $n=29$ participants [103]. The observed days to relapse had an estimated coefficient of variation $=0.40$. Preliminary evidence suggests a mean $=20$ days to relapse in the sham groups $(n=11)$. The decision to include a control group improves the internal validity and confidence in effect attribution [98]. Because control groups impact feasibility (e.g., cost and size), we chose a factorial design in which two dosing components are simultaneously examined and reducing the size of the control group to the number needed to maintain precision, power, and the anticipated effect size. We estimate that we will be able to maintain estimated power and precision to detect the anticipated effect size with $n=11$ in the sham control conditions (34\% of the $\mathrm{n}$ for the active condition), for a total sample size of $n=258$. Preliminary studies suggest that $14 \%$ of those we recruit will not attend any stimulation sessions and we plan to recruit $n=300$ as a result.

\section{Participants}

This study employs direct, community-based efforts focused on social marketing methods and small media (e.g., flyers in local businesses, advertisements in free local newspapers, talks with community groups). We aim to enroll $n=258$ participants who receive at least one rTMS session. We anticipate that this study will enroll 50\% women and 30\% Black or African American smokers. Women and Black smokers make up a smaller proportion of the smoking population than White men, but women and Black smokers tend to be underrepresented in many clinical trials and suffer disproportionately from tobacco-related disease. The population in Erie county, the county in which Roswell Park Cancer Institute is located, is about 14\% Black [104].

To be included in this study, participants must meet the following criteria: be a healthy, right-handed adult, age 18-65 years old, negative urine drug and pregnancy test at the in-person screening assessment, able to read at the 8th grade level, pass the Transcranial Magnetic Stimulation Adult Safety and Screening Questionnaire [105], smoke between 5 and 25 cigarettes per day, and intend to quit smoking in the next 30 days. Participants with the following characteristics will be excluded: personal history of epilepsy; anticonvulsant medication use; current complications from head injury; history of aneurysm, stroke, or cranial neurosurgery; active 
diagnosis of migraine headaches, major depressive disorder, bipolar disorder, or schizophrenia-spectrum disorder; clinically significant findings on a magnetic resonance imaging (MRI; e.g., tumor, aneurysm, ischemic changes, etc.); metal implants or neurostimulators in the head, neck, or cochlea; a pacemaker; currently taking medications that lower seizure threshold or for tobacco cessation; are pregnant or planning to become pregnant in the next 6 months; currently use forms of tobacco other than cigarettes; score above 48.3 on the Claustrophobia Questionnaire at the baseline assessment (to ensure participants can undergo the MRI of the head); or are unwilling or unable to follow protocol requirements.

\section{Equipment}

Stimulation will be delivered with the Magstim Super RAPID $^{2}$ PLUS1 System with Magstim $70 \mathrm{~mm}$ Double Air Film Active and Sham Figure of 8 Coils to deliver active and sham rTMS. Focal electrical stimulation will be delivered with a DS3 Isolated Stimulator (Digitimer Ltd., Welwyn Garden City, Herforshire, U.K.). Electrical pulses will be triggered by the TMS controller so that each pulse coincides precisely with the clicking sounds of the sham coil. The Brainsight for TMS Neuronavigational system (Rouge Research, Inc.) will be used for precise placement of rTMS coils.

\section{Procedures}

Participants undergo extensive screening over the telephone prior to being invited to an in-person interview during which urine drug and pregnancy tests are administered. After providing informed consent, participants are administered a baseline assessment and undergo an MRI of the head (1.5 or 3 Tesla, no contrast, slice spacing $1 \times 1 \times 1 \mathrm{~mm}$ ), which is read by a radiologist. If the images reflect no contraindications for participation, participants are randomized and scheduled for the quit date, quit date counseling, and their regimen of rTMS sessions. See Table 1 for a summary of the study measures. The average time from baseline to first rTMS session is 30 days $(\mathrm{SD}=20.4)$.

Participants receive reminder calls over the phone prior to the baseline, MRI, and all outcome assessments. Prior to the first rTMS session, quit counseling occurs over the phone and provides another opportunity to remind them of their appointments. In person, at the first rTMS session, study staff provide participants with a calendar showing all study visits. Study staff reiterate the importance of attending all rTMS sessions and calling if they are experiencing any study-related difficulties, including attending sessions. For the duration of the trial, participants are asked to refrain from the use of any smoking cessation aids, including nicotine replacement therapy in all forms as well as bupropion and varenicline.

\section{Stimulation site targeting}

The dlPFC is a functional node in the PFC that lies in the middle of the frontal gyrus in the lateral part of Brodmann's area 9 near 46 [119]. Prior to undergoing the MRI, we use the extended International 10-10 scalp electrode system to place a vitamin E capsule at the AF3 electrode position on the head to establish a fiducial marker for the stimulation site. The dIPFC is located between the F3 and the AF3 electrode positions [120]. The 10-10 system accounts for the curvilinear nature of the head, variability in head size, and some inter-session variability associated with other approaches [120]. The cognitive functions of interest are in the anterior region of the dlPFC [119] suggesting that the AF3 electrode position is the ideal site of stimulation. These procedures for achieving precision are highly consistent with current practice and recommendations [120,121]. When the MRI of the head is uploaded into the Brainsight (Rogue Research, Inc) neuro-navigation system the fiducial marker is apparent on the image.

\section{Randomization}

Random sequence allocation software is used to assign participants to treatment conditions which are concealed via password protected files until intervention group is assigned. To reduce inter-group variability, we use permuted block, stratified randomization by dependence level using the Fagerstrom test for nicotine dependence (FTND) score (low <4, high $\geq 4$ ). Nicotine dependence level significantly influences risk of relapse [122] and influenced outcomes in the feasibility study [94] and a pooled analysis of varenicline studies [123]. Project coordinators are the only individuals on the team who have access to the random assignment of participants. All other study and investigative team members, including technicians who administer rTMS and interviewers who assess outcomes, are blind to study assignment. Breaking the blinding code should only occur in situations when knowledge of the treatment is essential for participant care (e.g., due to severe adverse events (SAEs)). If unblinding is necessary, the already-unblinded project coordinator should work directly with the study physician rather than unblinding team members. Unblinding should not necessarily be a reason for discontinuation.

\section{Quit Day}

The quit day is the day immediately prior to the first rTMS session. Participants are provided with psychoeducational materials and $30 \mathrm{~min}$ of brief structured counseling over the telephone at least 3 days prior to the quit attempt to help them achieve the initial $24 \mathrm{~h}$ of 
Table 1 Study measures

\begin{tabular}{|c|c|c|c|c|c|c|}
\hline & $\begin{array}{l}\text { Phone } \\
\text { Screening }\end{array}$ & $\begin{array}{l}\text { In-Person } \\
\text { Interview }\end{array}$ & $\begin{array}{l}\text { Baseline } \\
\text { Assessment }\end{array}$ & $\begin{array}{l}1 \mathrm{st} \\
\text { TMS }\end{array}$ & $\begin{array}{l}\text { All other TMS } \\
\text { sessions }\end{array}$ & $\begin{array}{l}\text { Outcome } \\
\text { Assessments }\end{array}$ \\
\hline Race, ethnicity, age & $x$ & & & & & \\
\hline Medication list (self-report) & $x$ & $x$ & & $x$ & & \\
\hline TMS Safety and Screening [105] & $x$ & $x$ & & & & \\
\hline Urine drug test & & $x$ & & & & \\
\hline Urine pregnancy test & & $x$ & & & & \\
\hline Claustrophobia questionnaire [106] & & $x$ & & & & \\
\hline MRI (Sagittal T1 Axial 3-D SPGR) & & & $x$ & & & \\
\hline Complete demographic information & & & $x$ & & & $x$ \\
\hline Carbon monoxide level & & & $x$ & $x$ & & $x$ \\
\hline 5-Trial adjusting delay discounting task $(\$ 100, \$ 1000)[107]$ & & & $x$ & & & $x$ \\
\hline MacArthur scale of subjective social status [108] & & & $x$ & & & $x$ \\
\hline Motivation to quit ${ }^{a}$ & & & $x$ & $x$ & $x$ & $x$ \\
\hline Self-efficacy to quit ${ }^{a}$ & & & $x$ & $x$ & $x$ & $x$ \\
\hline Treatment engagement ${ }^{\mathrm{a}}$ & & & $x$ & $x$ & $x$ & $x$ \\
\hline Barratt impulsiveness scale-11 [109] & & & $x$ & & & $x$ \\
\hline Time perspective questionnaire [110] & & & $x$ & & & $x$ \\
\hline Perceived stress scale -4 [111] & & & $x$ & $x$ & $x$ & $x$ \\
\hline Positive and negative affect scale [112] & & & $x$ & $x$ & $x$ & $x$ \\
\hline Center for epidemiologic studies depression scale [113] & & & $x$ & $x$ & $x$ & $x$ \\
\hline State-trait anxiety inventory [114] & & & $x$ & $x$ & $x$ & $x$ \\
\hline Cognitive-behavioral therapy skills questionnaire [115] & & & $x$ & & & $x$ \\
\hline Perceived research burden) [116]. & & & $x$ & & & $x$ \\
\hline Side effects & & & & $x$ & $x$ & \\
\hline Booklet content tracking & & & & $x$ & $x$ & $x$ \\
\hline Blinding questions (participant and staff) [117]. & & & & $x$ & $x$ & \\
\hline Timeline follow-back daily cigarette use [118] & & & & & & $x$ \\
\hline
\end{tabular}

${ }^{\mathrm{a}}$ Measured on a scale of $0-10$ where $0=$ not at all and $10=$ the most possible

abstinence. Participants must abstain from smoking for at least $24 \mathrm{~h}$ as evidenced by an expired breath carbon monoxide level $10 \mathrm{ppm}$ or less immediately prior to the first stimulation session [122]. Expired breath Carbon Monoxide (CO) levels of 8-10 ppm are recommended by the SRNT Subcommittee on Biochemical Verification as a cut-off for abstinence [122]. The half-life of $\mathrm{CO}$ varies by activity level, but can be as long as $8 \mathrm{~h}$ [122]. We use this cut-off for initial abstinence because higher $\mathrm{CO}$ levels, such as $40-50 \mathrm{ppm}$, are unlikely to reach lower than $10 \mathrm{ppm}$ within $24 \mathrm{~h}$ among sedentary smokers who might have compromised respiratory systems [122]. Participants with expired breath $\mathrm{CO}$ levels greater than 10 ppm are encouraged to try again but do not initiate stimulation until they achieve $24 \mathrm{~h}$ of abstinence. Although 24-h abstinence is required for the first session to establish a valid quit attempt, abstinence is not required for receiving the remaining rTMS sessions.

\section{Preparation for rTMS}

All participants are prepared identically. Individuals' brains have varying levels of sensitivity to TMS pulses due to a variety of factors (thickness of skin and skull bone, space between scalp and brain tissue, etc.). The Motor Threshold (MT) is a well-established method of standardizing the stimulator impact on brain tissue. While seated in the stimulation chair, 3 electrodes are placed over the abductor pollicis brevis muscle (APB) on the hand. The MT is defined as the minimum stimulation intensity required to elicit a motor evoked potential of $50 \mu \mathrm{V}$ from the APB in 3 of 6 trials. Once participants' MT is determined, the stimulator is set to deliver stimulation at $110 \%$ of the MT. Once the MT has been determined, the rTMS technician and the participant leave the room and the project coordinator attaches either the active or sham coil as per random assignment and documents and double-checks accordingly. Once 
the appropriate coil is attached, the coordinator invites the technician and the participant to re-enter the room and preparations continue. The active and the sham coil are identical in appearance and set-up. Neither the participant nor the technician delivering stimulation will be able to tell a difference.

Prior to stimulation, conductive skin preparation gel is placed on two rectangular, carbon-impregnated rubber electrodes $(4 \times 5 \mathrm{~cm})$. The electrodes are placed firmly over the left frontalis muscle about $1 \mathrm{~cm}$ above the eyebrow underneath the headband that holds the Brainsight reflective tracking balls. The first electrode is aligned above the medial side of the eyebrow and the second aligned with the lateral aspect of the left eye. These electrodes deliver focal electrical stimulation in timing with the rTMS pulses as the sham technique but are not activated during the active rTMS condition.

\section{rTMS sessions}

The Brainsight neuro-navigation system is used to control the angle and distance of the coil relative to the curvilinear surface of brain so that proper positioning is achieved, maintained, and replicated across sessions. Each session provides 900 pulses of $20 \mathrm{~Hz}$ rTMS of the left dIPFC at $110 \%$ of the MT. Pulses are delivered in 45 20-pulse trains of $1 \mathrm{~s}$ duration with an inter-train interval of $20 \mathrm{~s}$. The actual stimulation time is $16 \mathrm{~min}$. Participants read the 8 booklets, in order, during the first 8 days of stimulation. The booklets are added one-by-one to a packet which includes all the materials necessary for tracking content exposure. Participants take the packet home with them after sessions. Following each session, participants complete a post-session assessment.

\section{Outcome assessment}

Participants are followed for 24weeks after initiating rTMS. Number of cigarettes smoked per day is assessed every two weeks by telephone with the Timeline Follow Back (TLFB) procedure [124]. In-person outcome assessments are conducted $4,8,12,18$, and 24 weeks after first rTMS session. CO in exhaled breath is used to biochemically validate abstinence during in-person outcome assessment visits. CO levels of $\leq 5 \mathrm{ppm}$ will be considered abstinent from smoking [122]. All participants who begin rTMS are invited to complete their OAs.

\section{Discontinuing participants}

Participants may choose to end study involvement at any time and will not be replaced after starting their scheduled rTMS sessions. Any missed sessions are noted and participants are welcome to continue their treatment as scheduled (i.e., as they would had the sessions not been missed). We are unable to reschedule any
rTMS sessions that fall outside of the 4-sessions in 7days window.

\section{Data analysis plan}

Data will be analyzed at the end of the trial by the authors, including a project coordinator who is unblinded to study condition. With its wealth of resources, including biostatisticians, RPCC is well suited to assisting with any statistical concerns and a Data Monitoring Committee was not deemed necessary. In the feasibility study, the median and mean latency to relapse was 33.5 days and 45.2 days in the active condition, and 8 days and 20.5 days in the sham condition (RR 0.29, CI 0.10-0.76, Likelihood ratio $X^{2}$ with $1 \mathrm{df}=6.40, p=.01$ ). Assuming days to relapse are exponentially distributed, the corresponding hazard ratio (active vs. sham) in the feasibility study was between 0.24 (based on medians) and 0.45 (based on means), a small to medium effect size but significant in the context of a study with $n=29$ participants [103], and the observed days to relapse had an estimated coefficient of variation $=0.40$. This suggests a mean of 20 days to relapse in the sham groups $(n=11)$ in that study. With $n=32$ in the active groups and a common standard deviation of $20 \times 0.40=8.0$ across cells, this design has $80 \%$ power to detect a main effect of at least 4 days to relapse in a dichotomous factor (Cohen's $d=.50$ ) (2-sided alpha $=0.05, \mathrm{SD}=8.0)$. With exponentially distributed days to relapse, a 4-day change in the mean equates to a detectable hazard ratio of 0.85 or less, in favor of the active condition. Please note, the factorial design reflected in Fig. 1 should not be considered a multiple arm randomized control trial (RCT) $[125,126]$. Following recommendations from Collins et al., the sample size was estimated using smallest estimated effect size derived from the feasibility study (8 daily sessions in 14 days) $[94,125,126]$.

Data is stored securely at the study site using both electronic and paper sources. Participant's information will not be released outside of the study except as required for monitoring by the NIH or RPCC's Clinical Research Services (CRS) department. All rTMS-related data is stored on paper source forms and is manually entered into SPSS using double data entry to ensure data quality. The battery of psychological assessments at baseline and outcome visits is stored electronically and automatically scored. Assessments are listed in full in Table 1 and references are provided showing reliability and validity for each measure. Scoring was coded and tested prior to the first participant and will be scored as a secondary check using SPSS syntax after study completion. All data exists on Roswell Park's secure system, protected by the campus' firewalls. The project coordinator will oversee intra-study data sharing with the guidance of the PI. To maintain confidentiality, all identifying 
information is blinded in the final dataset and the dataset is not shared with anyone outside the study team. Participation is monitored via status notification updates submitted to RPCC's CRS. The CRS department is responsible for trial auditing at their discretion and the study team performs data quality control checks weekly. Results will not be communicated to participants directly but will be posted on ClinicalTrials.gov and published in a relevant journal agreed upon by all authors.

Prior to hypothesis testing, descriptive analyses will be conducted on all measures using appropriate summary statistics (i.e., mean and standard deviations, medians and interquartile range, and proportions). We anticipate $10-15 \%$ of participants will be lost to follow-up (LTF) after the first stimulation session. Initial analyses will examine treatment adherence and associations with missing data to determine whether missing data and censoring are non-informative (i.e., unrelated to the study). Sensitivity analyses will examine assumptions garnered from initial analyses and LTF will be examined as a study endpoint. Missing point prevalence outcome data will be addressed by imputing continued smoking for those LTF (i.e., intention to treat) and supplemented with multiple imputation analyses. All multivariate models will be computed using effect coding. Effect coding allows direct, independent estimation of main and interaction effects with $95 \%$ confidence intervals from a single model [127, 128]. Analyses will be conducted with and without adjusting for biologic variables (age, sex/ gender) and race. All multivariate models will be specified to include effect-coded covariates for the duration (3 levels, ref.: 8 days), the intensity (ref: $1 x$ per day) and the activity type (active, ref.: sham) and interactions to the 3rd order adjusting for biologically relevant covariates including the stratification factor. Multivariable adjustment generally improves the efficiency of analyses and avoids estimation bias from covariate imbalance. We will report outcomes appropriately quantified for the type of model used including hazard ratios, odds ratios, 95\% confidence intervals, and $p$-values.

\section{Aim one}

To examine the effects of stimulation duration and intensity on latency to relapse, point prevalence abstinence rates, delay discounting rates, cognitive-behavioral skills acquisition, and potential undesirable effects (e.g., noncompliance, participant burden, side effects) among smokers $(n=258)$ motivated to quit. Hypothesis 1: Duration and intensity will be positively associated with effect size for latency to relapse, abstinence rates, delay discounting rates, and cognitive-behavioral skills acquisition. We will use Cox proportional-hazards $(\mathrm{CPH})$ models to examine latency to relapse outcomes. $\mathrm{CPH}$ models are appropriate for time-to-event data that include censored values $[129,130]$. Data from participants who relapse are directly observed (i.e. not censored). Data from participants who are LTF or who do not relapse are censored on their date of last follow-up [131]. Ties in failure times will be handled using methods developed by Efron [132]. We will use logistic regression models to examine binary 12 and 24-week point prevalence abstinence. We will use repeated measures Generalized Linear models to examine delay discounting and cognitive-behavioral skills acquisition. Hypothesis 2: The highest duration and intensity doses will be associated with greater levels of rTMS noncompliance due to subject burden. We will examine the undesirable effects of duration and intensity using a repeated measures Generalized Linear model.

\section{Aim two}

We will identify the most promising dosing regimen by systematically balancing efficacy and undesirable effects. Non-compliance is a primary focus of these analyses because noncompliance (i.e., non-adherence to session attendance) often emerges from multiple undesirable effects, including participant burden and side effects. To ensure the feasibility of a dosing regimen for efficacy testing, the proportion of participants who complete the regimen should be a least $45 \%[133,134]$. Based on preliminary work $[10,94]$, we expect at least $75 \%$ of participants to complete assigned stimulation sessions across conditions, with variability among conditions. Active dosing regimens with completion rates below $45 \%$ will be deemed intolerable and excluded from further consideration. For the remaining groups, selection of the most promising dosing regimen will be based on effect size and 95\% confidence limit estimates, relative to appropriate controls and the impact of undesirable effects. Groups with overlapping confidence intervals will be considered equivalent, and the less burdensome treatment will be preferred.

\section{Adverse events}

For this study, adverse events are monitored each visit by asking participants how they are feeling. Participants are encouraged to describe any differences they notice since the previous day and immediately following rTMS. All adverse events are recorded and those that meet the criteria for a serious adverse event (SAE) will be reported to CRS and the IRB. The investigators define SAE as any medical event believed to be causally linked to rTMS treatment and results in the following: a threat to the life of a participant; severe or permanent disability; or prolonged hospitalization. While the trial does not have ancillary care insurance, RPCC's patient advocacy program functions as participant advocates as well and will be able to assist participants who experience 
adverse events related to study participation. Participants are advised of their services during the consenting process and would be reminded in the event of an adverse event. We have not experienced any SAEs as of the date of this publication. Unless the investigators feel rTMS or the study have caused them, SAEs that are noted after a participant completes or discontinues from the study will not be reported.

\section{Preliminary participant characteristics}

From October 2018 to January 2020, 503 people were screened for enrollment, $n=93$ (18.5\%) met initial eligibility requirements; of those, $n=59$ (63\%) initiated rTMS sessions and were fully enrolled. The participants recruited to date range in age from 20 to $65(M=50.2$, $\mathrm{SD}=10.6) ; 62.4 \%$ identify as female; and $70 \%$ identify as White or Caucasian, 23\% as Black or African American, $2 \%$ as American Indian/Alaska Native, $1 \%$ as Asian or Pacific Islander, and $4 \%$ as other or multi-ethnic. Half of the participants are partnered. The average FTND score is 4.5 and the average number of cigarettes per day $(\mathrm{CPD})$ is $14.57(\mathrm{SD}=6.07)$. Of those that have completed their rTMS regimen $(n=58), 88 \%$ percent have completed all their assigned rTMS sessions across conditions to date.

\section{Discussion}

This clinical trial integrates existing theoretical concepts, approaches, and methodologies from neuropsychology, behavioral economics, brain stimulation, clinical psychology, and the evidence-based treatment of tobacco dependence in the development of a promising and innovative approach to treat tobacco dependence. This study will establish an optimal dosing regimen for efficacy testing, the next essential next step in the development of this approach. The findings will significantly advance the research in the clinical application of rTMS as a treatment for tobacco dependence and perhaps other addictive disorders. In its early stages, this study is making progress in recruiting a relatively diverse group of participants. The percent of participants who complete their rTMS regimen as assigned across conditions is also promising and bodes well for future efficacy testing.

\section{Strengths and limitations}

A significant strength of this study is the double-blinded, randomized, sham-controlled factorial design. Randomization and double blinding protect against bias and support the rigor of the approach while the factorial design parsimoniously screens for duration and intensity simultaneously. The sham control allows for a comparison group to calculate effect sizes while accounting for non-specific treatment-related effects. Another significant strength lies in the rigor and depth and breadth of the outcomes which assess a spectrum of the proposed cognitive and behavioral influences of rTMS. The TLFB assessment will provide a detailed continuous abstinence outcome appropriate for the $\mathrm{CPH}$ survival analysis, but will also provide information about the timing of relapses, the frequency of lapses, and whether participants recover from a relapse and regain continued abstinence for secondary analyses. The outcomes also include repeated assessment of delay discounting rates which will provide information about the pattern of delay discounting rates, an indicator of the relative balance of activity in the CNDS model across time. Important strengths also include the sample size, which provides enough power to confidently examine differences among all the outcome variables, and use of the $\mathrm{CPH}$ models which allow the inclusion of data from all participants who initiate treatment without imputation. We also see the intended representation of $50 \%$ women and $30 \%$ Black smokers in this study as a significant strength in terms of the generalizability of the findings.

This study has some potential limitations as well. This study is ambitious. While we have carefully evaluated our recruitment goals, our resources, our combined expertise, and the ideal study sample, and can demonstrate that the scope of this study is achievable in the 5-year timeframe, the recruitment goals remain ambitious and we estimate that it will require $75-85 \%$ capacity of our systems, which are open for scheduling from 8 am to 8 pm Monday-Friday. Thus, there is little leeway for accommodating unforeseen circumstances like equipment break-downs, staff illness/turnover, and weather emergencies. In addition, there is the chance that the treatment we ultimately develop might not be cost-effective. However, in order to calculate cost-effectiveness, we must first determine optional dosing and efficacy. This limitation, however, might be ameliorated in the future given the range of health care providers who are able to deliver rTMS has increased dramatically since 2013. In concert, we expect the cost to continue to decrease in the next several years. Once dosing and efficacy are determined, we can determine cost per quit and quality of life years saved to determine cost-effectiveness. Recent evidence suggests that rTMS treatment for depression is more cost-effective than anti-depressant therapy $[135,136]$. Finally, the design relies on smokers to attain $24 \mathrm{~h}$ of abstinence prior to stimulation and this might be too difficult or too discouraging for some participants. While attrition prior to the first session of any treatment for tobacco dependence is common and in the feasibility study participants reported that scheduling and other issues caused non-attendance, we suspect that the inability to achieve $24 \mathrm{~h}$ of abstinence contributed to a lack of compliance in the feasibility study. For this study, we revised our approach to provide standardized counseling and assistance at least 3 days prior to the quit attempt to help participants plan for and be 
successful with their 24-h quit. As in the feasibility study, participants with expired breath CO levels $>10 \mathrm{ppm}$ will be encouraged to try again, but will not initiate stimulation until they achieve $\leq 10 \mathrm{ppm}$. In defense of this requirement, which is intended to standardize the quit date and support a valid quit attempt, over half of smokers attempt to quit every year and the vast majority of these attempts last $>24 \mathrm{~h}[3,137]$.

In conclusion, the findings from this study are expected to have a sustained and powerful influence on advancing this novel approach to treating tobacco dependence. This study is the next logical step in the development of a new approach for preventing relapse to smoking, which was previously found to be feasible and to have potential long-term efficacy. The findings will inform future research on clinical approaches that combine rTMS with other evidence-based treatments for tobacco dependence and perhaps other addictions.

\section{Abbreviations \\ APB: Abductor pollicis brevis; CNDS: Competing Neurobehavioral Decision Systems; CLQ: Claustrophobia questionnaire; CO: Carbon monoxide; CRS: Clinical research services; dIPFC: Dorsolateral prefrontal cortex; FTND: Fagerstrom test for nicotine dependence; HF: High frequency; IRB: Institutional Review Board; LTF: Lost to follow-up; LTP: Long-term potentiation; MT: Motor threshold; MRI: Magnetic resonance imaging; PFC: Prefrontal cortex; rTMS: Repetitive transcranial magnetic stimulation; SAE: Serious adverse event}

\section{Acknowledgements}

Not applicable.

\section{Authors' contributions}

EC developed and drafted this manuscript. CES, WKB, and MM were involved in the conception and design of this study. CR developed the study protocol. EC, AL, and $A Q$ refined the study protocol. RA and $A B$ developed and refined the imaging protocols and read all the MRIs. CES and MM provide final approval for eligibility. All authors contributed to the critical review of the manuscript. The authors read and approved the final manuscript as well as the choice of submission journal.

\section{Funding}

This study is paid for by the National Institutes of Health, National Cancer Institute (R01CA229415) which have no financial interest in its outcome. This work was also supported by Roswell Park Comprehensive Cancer Center and National Cancer Institute (NCl) grant P30CA016056. The funding agents do not have an active role in study design, collection, management, analysis, or interpretation of data, report writing, or the decision to submit publications or reports. The funding body peer reviewed the study protocol.

\section{Availability of data and materials}

The datasets generated and/or analyzed during the current study are not publicly available due to the ongoing nature of the study but will be available from the corresponding author upon reasonable request.

\section{Ethics approval and consent to participate}

Ethics approval has been obtained by Roswell Park Comprehensive Cancer Center's Institutional Review Board (I-65718). Participants do not qualify as a vulnerable population as defined by the protection guidelines (i.e., not minors, pregnant women, under legal coercion or restriction, or mentally impaired). Participants will be competent and able to legally provide voluntary written informed consent. The consent process will inform participants in the detail of the procedures, time, involvement, compensation, risks, and treatment options other than participation in the study. Participants will also be instructed that they can withdraw from participation at any time without losing any of the compensation that they have earned to that point and that enrollment in the study will in no way adversely affect the provision of treatment elsewhere (e.g., Quitline, Roswell Park, etc.)

\section{Consent for publication}

Not applicable.

\section{Competing interests}

The authors declare that they have no competing interests. The study received external funding from the National Institutes of Health, National Cancer Institute, as noted below.

\section{Author details}

${ }^{1}$ Roswell Park Comprehensive Cancer Center, Buffalo, NY 14203, USA. ${ }^{2}$ Fralin Biomedical Research Institute at Virginia Tech Carilion, Roanoke, VA 24016, USA.

Received: 18 March 2020 Accepted: 5 April 2020

Published online: 01 May 2020

\section{References}

1. (DHHS) USDoHaHS. The health consequences of smoking - 50 years of progress. A report of the surgeon general. In: Services DoHaH, editor. Atlanta: U.S. Department of Health and Human Services, Centers for Disease Control and Prevention, National Center for Chronic Disease Prevention and Health Promotion, Office on Smoking and Health; 2014.

2. (ACS) ACS. Cancer facts and figures 2016. Atlanta: American Cancer Society; 2016.

3. Babb S, Malarcher A, Schauer G, Asman K, Jamal A. Quitting smoking among adults - United States, 2000-2015. MMWR Morb Mortal Wkly Rep. 2017;65(52):1457-64.

4. Malarcher A, Dube S, Shaw L, Babb S, Kaufmann RB. Quitting Smoking Among Adults --- United States, 2001--2010. Morb Mortal Wkly Rep. 2011; 60(44):1513-9.

5. Fiore MC, Jaén CR, Baker TB, Bailey WC, Benowitz NL, Curry SJ, et al. Treating tobacco use and dependence: 2008 Update. Clinical practice guideline. In: Services UDoHaH, editor. . Rockville: Public Health Service; 2008.

6. Hughes JR. Effects of abstinence from tobacco: valid symptoms and time course. Nicotine Tob Res. 2007;9(3):315-27.

7. Heyman GM. Addiction: a disorder of choice. Cambridge: Harvard University Press; 2009.

8. Levasseur-Moreau J, Fecteau S. Translational application of neuromodulation of decision-making. Brain Stimul. 2012;5(2):77-83.

9. Kim S, Lee D. Prefrontal cortex and impulsive decision making. Biol Psychiatry. 2011;69(12):1140-6.

10. Sheffer CE, Mennemeier M, Landes RD, Bickel WK, Brackman S, Dornhoffer J, et al. Neuromodulation of delay discounting, the reflection effect, and cigarette consumption. J Subst Abuse Treat. 2013;45(2):206-14.

11. Edwards S, Koob GF. Neurobiology of dysregulated motivational systems in drug addiction. Future Neurol. 2010;5(3):393-401.

12. Botvinick MM, Braver TS, Barch DM, Carter CS, Cohen JD. Conflict monitoring and cognitive control. Psychol Rev. 2001;108(3):624-52.

13. Baddeley A. Working memory: looking back and looking forward. Nat Rev Neurosci. 2003;4(10):829-39.

14. Brody AL, Mandelkern MA, London ED, Childress AR, Lee GS, Bota RG, et al. Brain metabolic changes during cigarette craving. Arch Gen Psychiatry. 2002;59(12):1162-72

15. McBride D, Barrett SP, Kelly JT, Aw A, Dagher A. Effects of expectancy and abstinence on the neural response to smoking cues in cigarette smokers: an fMRI study. Neuropsychopharmacology. 2006;31(12):2728-38.

16. Bechara A. Decision making, impulse control and loss of willpower to resist drugs: a neurocognitive perspective. Nat Neurosci. 2005;8(11):1458-63.

17. Ernst M, Paulus MP. Neurobiology of decision making: a selective review from a neurocognitive and clinical perspective. Biol Psychiatry. 2005;58(8): 597-604.

18. Evans JS. Dual-processing accounts of reasoning, judgment, and social cognition. Annu Rev Psychol. 2008;59:255-78.

19. Fecteau S, Fregni F, Boggio PS, Camprodon JA, Pascual-Leone A. Neuromodulation of decision-making in the addictive brain. Subst Use Misuse. 2010;45(11):1766-86. 
20. Koob GF, Arends MA, Michel LM. Drugs, addiction, and the brain. Oxford: Elsevier; 2014.

21. Day AM, Kahler CW, Metrik J, Spillane NS, Tidey JW, Rohsenow DJ. Working memory moderates the association between smoking urge and smoking lapse behavior after alcohol Administration in a Laboratory Analogue Task. Nicotine Tob Res. 2015;17(9):1173-7.

22. Lerman C, Gu H, Loughead J, Ruparel K, Yang Y, Stein EA. Large-scale brain network coupling predicts acute nicotine abstinence effects on craving and cognitive function. JAMA Psychiat. 2014;71(5):523-30.

23. Xu J, Mendrek A, Cohen MS, Monterosso J, Rodriguez P, Simon SL, et al. Brain activity in cigarette smokers performing a working memory task: effect of smoking abstinence. Biol Psychiatry. 2005;58(2):143-50.

24. Ernst M, Heishman SJ, Spurgeon L, London ED. Smoking history and nicotine effects on cognitive performance. Neuropsychopharmacology. 2001;25(3):313-9.

25. Koob GF, Le Moal M. Addiction and the brain antireward system. Annu Rev Psychol. 2008:59:29-53.

26. Koob GF, Le Moal M. Review. Neurobiological mechanisms for opponent motivational processes in addiction. Philos Trans R Soc Lond B Biol Sci. 2008;363(1507):3113-23.

27. McClure SM, Bickel WK. A dual-systems perspective on addiction: contributions from neuroimaging and cognitive training. Ann N Y Acad Sci. 2014;1327:62-78.

28. Bickel WK, Miller ML, Yi R, Kowal BP, Lindquist DM, Pitcock JA. Behavioral and neuroeconomics of drug addiction: competing neural systems and temporal discounting processes. Drug Alcohol Depend. 2007;90(Suppl 1): s85-91.

29. Bickel WK, Yi R. Temporal discounting as a measure of executive function: insights from the competing neuro-behavioral decision system hypothesis of addiction. Adv Health Econ Health Serv Res. 2008;20:289-309.

30. Koffarnus MN, Jarmolowicz DP, Mueller ET, Bickel WK. Changing delay discounting in the light of the competing neurobehavioral decision systems theory: a review. J Exp Anal Behav. 2013;99(1):32-57.

31. Hanlon CA, Dowdle LT, Austelle CW, DeVries W, Mithoefer O, Badran BW, et al. What goes up, can come down: Novel brain stimulation paradigms may attenuate craving and craving-related neural circuitry in substance dependent individuals. Brain Res. 2015;1628(Pt A):199-209.

32. McClure SM, Laibson DI, Loewenstein G, Cohen JD. Separate neural systems value immediate and delayed monetary rewards. Science. 2004;306(5695):503-7.

33. Alexander GE, DeLong MR, Strick PL. Parallel organization of functionally segregated circuits linking basal ganglia and cortex. Annu Rev Neurosci. 1986;9:357-81.

34. Mackillop J, Kahler CW. Delayed reward discounting predicts treatment response for heavy drinkers receiving smoking cessation treatment. Drug Alcohol Depend. 2009;104(3):197-203.

35. Bickel WK, Koffarnus MN, Moody L, Wilson AG. The behavioral- and neuroeconomic process of temporal discounting: A candidate behavioral marker of addiction. Neuropharmacology. 2014;76 Pt B:518-27.

36. Bickel WK, Mellis AM, Snider SE, Moody L, Stein JS, Quisenberry AJ. Novel therapeutics for addiction: behavioral and Neuroeconomic approaches. Curr Treat Options Psychiatry. 2016;3(3):277-92.

37. McClure SM, Ericson KM, Laibson DI, Loewenstein G, Cohen JD. Time discounting for primary rewards. J Neurosci. 2007;27(21):5796-804.

38. Vanderveldt A, Oliveira L, Green L. Delay discounting: pigeon, rat, human-does it matter? J Exp Psychol Anim Learn Cogn. 2016;42(2):141-62.

39. Odum AL. Delay discounting: I'm a k, you're a k. J Exp Anal Behav. 2011;96(3):427-39.

40. Kirby KN. Bidding on the future: evidence against normative discounting of delayed rewards. J Exp Psychol Gen. 1997;126:54-70.

41. Mazur JE. An adjusting procedure for studying delayed reinforcement. In: JEM MLC, Nevin JA, Rachlin H, editors. The Effect of Delay and of Intervening Events on Reinforcement Value, Quantitative Analyses of Behavior. 5th ed. Hillsdale: Erlbaum; 1987. p. 55-73.

42. Ainslie G. Specious reward: A behavioral theory of impulsiveness and impulse control. Psychol Bull. 1975;82:483-96.

43. Logue AW. Research on self-control: an integrating framework. Behav Brain Sci. 1988;11:665-709.

44. Bickel WK, Yi R, Kowal BP, Gatchalian KM. Cigarette smokers discount past and future rewards symmetrically and more than controls: is discounting a measure of impulsivity? Drug Alcohol Depend. 2008;96(3):256-62.

45. Baker F, Johnson MW, Bickel WK. Delay discounting in current and neverbefore cigarette smokers: similarities and differences across commodity, sign, and magnitude. J Abnorm Psychol. 2003;112(3):382-92.
46. Bickel WK, Madden GJ. A comparison of measures of relative reinforcing efficacy and behavioral economics: cigarettes and money in smokers. Behav Pharmacol. 1999;10(6-7):627-37.

47. Mitchell SH. Measures of impulsivity in cigarette smokers and non-smokers. Psychopharmacology. 1999;146(4):455-64.

48. Reynolds B. Do high rates of cigarette consumption increase delay discounting? A cross-sectional comparison of adolescent smokers and young-adult smokers and nonsmokers. Behav Processes. 2004;67(3):545-9.

49. Odum AL, Madden GJ, Bickel WK. Discounting of delayed health gains and losses by current, never- and ex-smokers of cigarettes. Nicotine Tob Res. 2002;4(3):295-303.

50. Yoon JH, Higgins ST, Heil SH, Sugarbaker RJ, Thomas CS, Badger GJ. Delay discounting predicts postpartum relapse to cigarette smoking among pregnant women. Exp Clin Psychopharmacol. 2007;15(2):176-86.

51. Krishnan-Sarin S, Reynolds B, Duhig AM, Smith A, Liss T, McFetridge A, et al. Behavioral impulsivity predicts treatment outcome in a smoking cessation program for adolescent smokers. Drug Alcohol Depend. 2007;88(1):79-82.

52. Sheffer CE, Mackillop J, McGeary J, Landes R, Carter L, Yi R, et al. Delay discounting, locus of control, and cognitive impulsiveness independently predict tobacco dependence treatment outcomes in a highly dependent, lower socioeconomic group of smokers. Am J Addict. 2012;21(3):221-32.

53. Sheffer CE, Christensen DR, Landes R, Carter LP, Jackson L, Bickel WK. Delay discounting rates: a strong prognostic indicator of smoking relapse. Addict Behav. 2014;39(11):1682-9.

54. Stanger C, Ryan SR, Fu H, Landes RD, Jones BA, Bickel WK, et al. Delay discounting predicts adolescent substance abuse treatment outcome. Exp Clin Psychopharmacol. 2012;20(3):205-12.

55. Ballard K, Knutson B. Dissociable neural representations of future reward magnitude and delay during temporal discounting. Neurolmage. 2009;45(1): $143-50$.

56. Mackillop J, Amlung MT, Wier LM, David SP, Ray LA, Bickel WK, et al. The neuroeconomics of nicotine dependence: a preliminary functional magnetic resonance imaging study of delay discounting of monetary and cigarette rewards in smokers. Psychiatry Res. 2012;202(1):20-9.

57. Bickel WK, Jarmolowicz DP, Mueller ET, Koffarnus MN, Gatchalian KM. Excessive discounting of delayed reinforcers as a trans-disease process contributing to addiction and other disease-related vulnerabilities: emerging evidence. Pharmacol Ther. 2012;134(3):287-97.

58. Fitzgerald PB, Fountain S, Daskalakis ZJ. A comprehensive review of the effects of rTMS on motor cortical excitability and inhibition. Clin Neurophysiol. 2006;117(12):2584-96.

59. Hoogendam JM, Ramakers GM, Di Lazzaro V. Physiology of repetitive transcranial magnetic stimulation of the human brain. Brain Stimul. 2010; 3(2):95-118.

60. Thickbroom GW. Transcranial magnetic stimulation and synaptic plasticity: experimental framework and human models. Exp Brain Res. 2007;180(4):583-93.

61. George MS, Nahas Z, Kozel FA, Li X, Denslow S, Yamanaka K, et al. Mechanisms and state of the art of transcranial magnetic stimulation. J ECT. 2002;18(4):170-81.

62. Di Lazzaro V, Pilato F, Saturno E, Oliviero A, Dileone M, Mazzone P, et al. Thetaburst repetitive transcranial magnetic stimulation suppresses specific excitatory circuits in the human motor cortex. J Physiol. 2005;565(Pt 3):945-50.

63. Hallett M. Transcranial magnetic stimulation and the human brain. Nature. 2000;406(6792):147-50.

64. Guse B, Falkai P, Wobrock T. Cognitive effects of high-frequency repetitive transcranial magnetic stimulation: a systematic review. J Neural Transm. 2010;117(1):105-22.

65. Li Y, Wang L, Jia M, Guo J, Wang H, Wang M. The effects of high-frequency rTMS over the left DLPFC on cognitive control in young healthy participants. PLoS One. 2017;12(6):e0179430.

66. Siebner HR, Rothwell J. Transcranial magnetic stimulation: new insights into representational cortical plasticity. Exp Brain Res. 2003;148(1):1-16.

67. Pascual-Leone A, Tormos JM, Keenan J, Tarazona F, Canete C, Catala MD. Study and modulation of human cortical excitability with transcranial magnetic stimulation. J Clin Neurophysiol. 1998;15(4):333-43.

68. Speer AM, Kimbrell TA, Wassermann EM. J DR, Willis MW, Herscovitch P, et al. opposite effects of high and low frequency rTMS on regional brain activity in depressed patients. Biol Psychiatry. 2000;48(12):1133-41.

69. Wu T, Sommer M, Tergau F, Paulus W. Lasting influence of repetitive transcranial magnetic stimulation on intracortical excitability in human subjects. Neurosci Lett. 2000;287(1):37-40. 
70. Perera T, George MS, Grammer G, Janicak PG, Pascual-Leone A, Wirecki TS. The clinical TMS Society consensus review and treatment recommendations for TMS therapy for major depressive disorder. Brain Stimul. 2016;9(3):336-46.

71. Figner B, Knoch D, Johnson EJ, Krosch AR, Lisanby SH, Fehr E, et al. Lateral prefrontal cortex and self-control in intertemporal choice. Nat Neurosci. 2010;13(5):538-9.

72. Cho SS, Ko JH, Pellecchia G, Van Eimeren T, Cilia R, Strafella AP. Continuous theta burst stimulation of right dorsolateral prefrontal cortex induces changes in impulsivity level. Brain Stimul. 2010;3(3):170-6.

73. Knoch D, Fehr E. Resisting the power of temptations: the right prefrontal cortex and self-control. Ann N Y Acad Sci. 2007;1104:123-34.

74. Knoch D, Gianotti LR, Pascual-Leone A, Treyer V, Regard M, Hohmann M, et al. Disruption of right prefrontal cortex by low-frequency repetitive transcranial magnetic stimulation induces risk-taking behavior. J Neurosci. 2006:26(24):6469-72.

75. Fecteau S, Knoch D, Fregni F, Sultani N, Boggio P, Pascual-Leone A. Diminishing risk-taking behavior by modulating activity in the prefrontal cortex: a direct current stimulation study. J Neurosci. 2007;27(46):12500-5.

76. Fecteau S, Pascual-Leone A, Zald DH, Liguori P, Theoret H, Boggio PS, et al. Activation of prefrontal cortex by transcranial direct current stimulation reduces appetite for risk during ambiguous decision making. J Neurosci. 2007;27(23):6212-8.

77. Rachid F. Neurostimulation techniques in the treatment of nicotine dependence: a review. Am J Addict. 2016;25(6):436-51.

78. Wing VC, Barr MS, Wass CE, Lipsman N, Lozano AM, Daskalakis ZJ, et al. Brain stimulation methods to treat tobacco addiction. Brain Stimul. 2012; 6(3):221-30.

79. Barr MS, Farzan F, Wing VC, George TP, Fitzgerald PB, Daskalakis ZJ. Repetitive transcranial magnetic stimulation and drug addiction. Int Rev Psychiatry. 2011;23(5):454-66.

80. Preston G, Anderson E, Silva C, Goldberg T, Wassermann EM. Effects of $10 \mathrm{~Hz}$ rTMS on the neural efficiency of working memory. J Cogn Neurosci. 2010;22(3):447-56.

81. Esslinger C, Schuler N, Sauer C, Gass D, Mier D, Braun U, et al. Induction and quantification of prefrontal cortical network plasticity using $5 \mathrm{~Hz}$ rTMS and fMRI. Hum Brain Mapp. 2014;35(1):140-51.

82. Chen AC, Oathes DJ, Chang C, Bradley T, Zhou ZW, Williams LM, et al. Causal interactions between fronto-parietal central executive and defaultmode networks in humans. Proc Natl Acad Sci U S A. 2013;110(49):19944-9.

83. Baeken C, De Raedt R. Neurobiological mechanisms of repetitive transcranial magnetic stimulation on the underlying neurocircuitry in unipolar depression. Dialogues Clin Neurosci. 2011;13(1):139-45.

84. Kravitz AV, Tomasi D, LeBlanc KH, Baler R, Volkow ND, Bonci A, et al. Corticostriatal circuits: Novel therapeutic targets for substance use disorders. Brain Res. 2015;1628(Pt A):186-98.

85. Cooke SF, Bliss TV. Plasticity in the human central nervous system. Brain. 2006;129(Pt 7):1659-73.

86. Bliss TV, Collingridge GL. A synaptic model of memory: long-term potentiation in the hippocampus. Nature. 1993;361(6407):31-9.

87. Serafini G, Pompili M, Belvederi Murri M, Respino M, Ghio L, Girardi P, et al. The effects of repetitive transcranial magnetic stimulation on cognitive performance in treatment-resistant depression. A systematic review. Neuropsychobiology. 2015;71(3):125-39.

88. Perkins KA, Conklin CA, Levine MD. Cognitive-behavioral therapy for smoking cessation: a practical guidebook to the most effective treatments. New York: Routledge; 2008

89. Abrams DB, Niaura R, Brown RA, Emmons KM, Goldstein MG, Monti PM. In: Barlow DH, editor. The tobacco dependence treatment handbook: a guide to best practices. New York: Guilford Press; 2003.

90. Beck AT, Rush AJ, Shaw BF, Emery G. Cognitive therapy for depression. New York: Guilford; 1979.

91. Vedeniapin A, Cheng L, George MS. Feasibility of simultaneous cognitive behavioral therapy and left prefrontal rTMS for treatment resistant depression. Brain Stimul. 2010;3(4):207-10.

92. Osuch EA, Benson BE, Luckenbaugh DA, Geraci M, Post RM, McCann U. Repetitive TMS combined with exposure therapy for PTSD: a preliminary study. J Anxiety Disord. 2009;23(1):54-9.

93. Miniussi C, Vallar G. Brain stimulation and behavioural cognitive rehabilitation: a new tool for neurorehabilitation? Neuropsychol Rehabil. 2011;21(5):553-9.
94. Sheffer CE, Bickel WK, Brandon TH, Franck CT, Deen D, Panissidi L, et al. Preventing relapse to smoking with transcranial magnetic stimulation: feasibility and potential efficacy. Drug Alcohol Depend. 2018;182:8-18.

95. Shiffman S, Shumaker SA, Abrams DB, Cohen S, Garvey A, Grunberg NE, et al. Models of smoking relapse. Health Psychol. 1986;5(Suppl):13-27.

96. Brandon $\mathrm{TH}$, Collins BN, Juliano LM, Lazev AB. Preventing relapse among former smokers: a comparison of minimal interventions through telephone and mail. J Consult Clin Psychol. 2000;68(1):103-13.

97. Marlatt G. Situational determinants of relapse and skill-training interventions. In: Marlatt G, Gordon J, editors. Relapse prevention. New York: Guilford Press; 1985.

98. Ting N. In: KK MG, Samet J, Tsiatis A, Wong W, editors. Dose finding in drug development. New York: Springer Science+business Media, Inc.; 2006.

99. Wassermann EM. Risk and safety of repetitive transcranial magnetic stimulation: report and suggested guidelines from the international workshop on the safety of repetitive transcranial magnetic stimulation, June 5-7, 1996. Electroencephalogr Clin Neurophysiol. 1998;108(1):1-16.

100. Rossi S, Hallett M, Rossini PM, Pascual-Leone A. Safety of TMSCG. Safety, ethical considerations, and application guidelines for the use of transcranial magnetic stimulation in clinical practice and research. Clin Neurophysiol. 2009;120(12):2008-39.

101. Teng S, Guo Z, Peng H, Xing G, Chen $H$, He B, et al. High-frequency repetitive transcranial magnetic stimulation over the left DLPFC for major depression: session-dependent efficacy: a meta-analysis. Eur Psychiatry. 2017:41:75-84.

102. Anderson B, Mishory A, Nahas Z, Borckardt JJ, Yamanaka K, Rastogi K, et al. Tolerability and safety of high daily doses of repetitive transcranial magnetic stimulation in healthy young men. J ECT. 2006;22(1):49-53.

103. Chinn S. A simple method for converting an odds ratio to effect size for use in meta-analysis. Stat Med. 2000;19(22):3127-31.

104. Bureau USC. QuickFacts: Erie County, New York; 2019.

105. Rossi S, Hallett M, Rossini PM, Pascual-Leone A. Screening questionnaire before TMS: an update. Clin Neurophysiol. 2011;122(8):1686.

106. Radomsky AS, Rachman S, Thordarson DS, Mclsaac HK, Teachman BA. The claustrophobia questionnaire. J Anxiety Disord. 2001;15(4):287-97.

107. Koffarnus MN, Bickel WK. A 5-trial adjusting delay discounting task: accurate discount rates in less than one minute. Exp Clin Psychopharmacol. 2014; 22(3):222-8.

108. Reitzel LR, Businelle MS, Kendzor DE, Li Y, Cao Y, Castro Y, et al. Subjective social status predicts long-term smoking abstinence. BMC Public Health. 2011;11:135.

109. Reise SP, Moore TM, Sabb FW, Brown AK, London ED. The Barratt impulsiveness Scale-11: reassessment of its structure in a community sample. Psychol Assess. 2013;25(2):631-42.

110. Sansone G, Fong GT, Hall PA, Guignard R, Beck F, Mons U, et al. Time perspective as a predictor of smoking status: findings from the international tobacco control (ITC) surveys in Scotland, France, Germany, China, and Malaysia. BMC Public Health. 2013;13(1):346.

111. Cohen S, Lichtenstein E. Perceived stress, quitting smoking, and smoking relapse. Health Psychol. 1990;9(4):466-78.

112. Watson D, Clark L, Tellegen A. Development and validation of brief measures of positive and negative affect. The PANAS scale. J Pers Soc Psychol. 1988:54:1063-70.

113. Radloff LS. The CES-D scale : a self-report depression scale for research in the general population. Appl Psychol Measur. 1977;1(3):385-401.

114. Spielberger CD. State-trait anxiety inventory (form Y). In: Hersen M, Bellack AS, editors. Dictionary of behavioral assessment techniques. New York: Pergamon; 1988. p. 448-50.

115. Jacob KL, Christopher MS, Neuhaus EC. Development and validation of the cognitive-behavioral therapy skills questionnaire. Behav Modif. 2011;35(6):595-618.

116. Lingler JH, Schmidt KL, Gentry AL, Hu L, Terhorst LA. A new measure of research participant burden: brief report. J Empir Res Hum Res Ethics. 2014;9(4):46-9.

117. James KE, Bloch DA, Lee KK, Kraemer HC, Fuller RK. An index for assessing blindness in a multi-Centre clinical trial: disulfiram for alcohol cessation--a VA cooperative study. Stat Med. 1996;15(13):1421-34.

118. Sobell LC, Sobell MB. Timeline follow-back: a technique for assessing selfconsumpton. In: Allen J, Litten RZ, editors. Measuring alcohol consumption: psychosocial and biological methods. Totowa: Humana Press; 1992. p. 41-72.

119. Cieslik EC, Zilles K, Caspers S, Roski C, Kellermann TS, Jakobs O, et al. Is there "one" DLPFC in cognitive action control? Evidence for heterogeneity from co-activation-based parcellation. Cereb Cortex. 2013;23(11):2677-89. 
120. Fitzgerald PB, Maller JJ, Hoy KE, Thomson R, Daskalakis ZJ. Exploring the optimal site for the localization of dorsolateral prefrontal cortex in brain stimulation experiments. Brain Stimul. 2009;2(4):234-7.

121. George MS, Taylor JJ, Short EB. The expanding evidence base for rTMS treatment of depression. Curr Opin Psychiatry. 2013;26(1):13-8.

122. verification $\mathrm{SSOB}$. Biochemical verification of tobacco use and cessation. Nicotine Tob Res. 2002;4(2):149-59.

123. Fagerstrom K, Russ C, Yu CR, Yunis C, Foulds J. The Fagerstrom test for nicotine dependence as a predictor of smoking abstinence: a pooled analysis of varenicline clinical trial data. Nicotine Tob Res. 2012;14(12):146773.

124. Toll BA, Cooney NL, McKee SA, O'Malley SS. Do daily interactive voice response reports of smoking behavior correspond with retrospective reports? Psychol Addict Behav. 2005;19(3):291-5.

125. Collins LM, Dziak JJ, Kugler KC, Trail JB. Factorial experiments: efficient tools for evaluation of intervention components. Am J Prev Med. 2014;47(4):498504.

126. Collins LM, Trail JB, Kugler KC, Baker TB, Piper ME, Mermelstein RJ. Evaluating individual intervention components: making decisions based on the results of a factorial screening experiment. Transl Behav Med. 2014;4(3):238-51.

127. Collins LM, Dziak JJ, Li R. Design of experiments with multiple independent variables: a resource management perspective on complete and reduced factorial designs. Psychol Methods. 2009;14(3):202-24.

128. Collins LM, Chakraborty B, Murphy SA, Strecher V. Comparison of a phased experimental approach and a single randomized clinical trial for developing multicomponent behavioral interventions. Clin Trials. 2009:6(1):5-15.

129. Hosmer DW, Lemeshow S. Applied survival analysis. New York: Wiley; 1999

130. Cox DR. Regresson models and life-tables. J R Stat Soc Ser B Methodol. 1972;34(2):187-220.

131. Shiffman S, Scharf DM, Shadel WG, Gwaltney CJ, Dang Q, Paton SM, et al. Analyzing milestones in smoking cessation: illustration in a nicotine patch trial in adult smokers. J Consult Clin Psychol. 2006;74(2):276-85.

132. Efron B, Tibshirani RJ. An introduction to the bootstrap. London. New York: Chapman \& Hall Ltd; 1993.

133. Bowen DJ, Kreuter M, Spring B, Cofta-Woerpel L, Linnan L, Weiner D, et al. How we design feasibility studies. Am J Prev Med. 2009;36(5):452-7.

134. Rounsaville BJ, Carroll KM, Onken LS. A stage model of behavioral therapies research: getting started and moving on from stage I Clinical Psychology. Sci Pract. 2001;8(2):133-42.

135. Nguyen KH, Gordon LG. Cost-effectiveness of repetitive transcranial magnetic stimulation versus antidepressant therapy for treatment-resistant depression. Value Health. 2015;18(5):597-604.

136. Simpson KN, Welch MJ, Kozel FA, Demitrack MA, Nahas Z. Cost-effectiveness of transcranial magnetic stimulation in the treatment of major depression: a health economics analysis. Adv Ther. 2009;26(3):346-68.

137. Hughes JR, Callas PW. Definition of a quit attempt: a replication test. Nicotine Tob Res. 2010;12(11):1176-9.

\section{Publisher's Note}

Springer Nature remains neutral with regard to jurisdictional claims in published maps and institutional affiliations.

Ready to submit your research? Choose BMC and benefit from:

- fast, convenient online submission

- thorough peer review by experienced researchers in your field

- rapid publication on acceptance

- support for research data, including large and complex data types

- gold Open Access which fosters wider collaboration and increased citations

- maximum visibility for your research: over $100 \mathrm{M}$ website views per year

At $\mathrm{BMC}$, research is always in progress.

Learn more biomedcentral.com/submissions 